\title{
The cost of synchronization
}

\author{
Synchronization of biochemical oscillators that are responsible for biological rhythms costs free energy. This \\ theoretical result suggests that part of the adenosine triphosphate molecules consumed by a Kai oscillator is \\ necessary for synchronization.
}

\section{Andre C. Barato}

B iochemical networks perform essential tasks for the functioning of living systems. They can be subjected to relatively large fluctuations and operate out of equilibrium, with the consumption of energy - often in the form of adenosine triphosphate (ATP) hydrolysis.

An interesting physical problem concerns the free-energy cost to maintain a biochemical network that performs a certain task. One such essential task is to display robust and precise biological rhythms such as circadian oscillations. This process relies on the synchronization of biochemical network oscillations and has been shown to require energy dissipation in a theoretical model developed by Dongliang Zhang and co-workers, as reported in Nature Physics ${ }^{1}$.

These oscillators are biochemical networks composed of interacting driven Brownian clocks. For instance, in the case of the Kai system found in cyanobacteria $^{2,3}$ - one of the best understood circadian clocks - the KaiC molecules play the part of the clocks. Synchronization is essential for the onset of oscillations in a network of clocks. Even though synchronization has been thoroughly studied in the well-established Kuramoto model of coupled oscillators ${ }^{4}$, its thermodynamic cost in biochemical oscillators is still unknown.

Zhang and co-workers have introduced a neat model for synchronization. A key feature of this model is the inclusion of exchange reactions, which change the phase of two clocks in a single reaction (Fig. 1). These exchange reactions are related to chemical interactions and can synchronize the clocks. This mechanism for synchronization differs from the Kuramoto model, which assumes a standard interaction energy between the clocks. The strength and frequency of the exchange reactions control the synchronization transition, such that the clocks synchronize if these parameters are large enough.
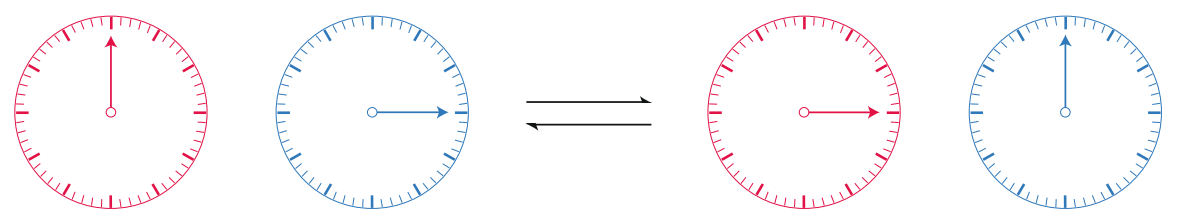

Fig. 1 | Illustration of the exchange reactions that modify the phase of two clocks. The phase of the clock corresponds to the phosphorylation level or conformational state of an individual molecule such as KaiC.

The model allowed for analytical calculations and the phase diagram was determined exactly.

Each individual clock is an out-ofequilibrium system that dissipates energy, as do the exchange reactions. The authors demonstrated that the thermodynamic rate of entropy production that quantifies energy dissipation can be decomposed into two terms: one related to reactions that change the phase of an individual clock and the other related to exchange reactions. Crucially, the authors found that the part of the energy dissipation that is associated with exchange reactions must be above a critical value for synchronization to occur. They also calculated the minimal amount of energy dissipation that is necessary to achieve a certain level of synchronization.

Besides this first exactly solvable model, Zhang and co-workers also analysed a model inspired by the Kai system. The results concerning the relation between synchronization and energy dissipation are similar to those obtained for the first model, which leads to the following interesting speculation: as the amount of hydrolysed ATP found per KaiC molecule in a period of oscillation is larger than the amount of ATP consumed in the cycles of all KaiC taken together, the extra ATP may be used to power synchronization.

This work opens the door for future experiments to confirm if ATP is indeed consumed for the synchronization in the Kai system, which would provide a beautiful connection between biophysics theory and experiment. The mechanism for synchronization in the model by Zhang and co-workers is different from the one in the Kuramoto model, and it would be interesting to investigate the differences and similarities of the synchronization transition between the two models. A better microscopic understanding of the model introduced by Zhang and co-workers, with a precise identification of the thermodynamic force that drives the cycles with exchange reactions, remains an open issue.

Unravelling the relation between energy dissipation and the performance of small biochemical systems is an ambitious goal. But a more complete picture of this relation can help us understand the evolution of biochemical networks and can provide us with recipes to build synthetic systems that can perform a biochemical task in an optimal way.

Andre C. Barato

Department of Physics, University of Houston, Houston, TX, USA.

e-mail:barato@uh.edu

Published online: 8 January 2020

https://doi.org/10.1038/s41567-019-0735-x

References

1. Zhang, D., Cao, Y., Ouyang, Q. \& Tu, Y. Nat. Phys. https://doi. org/10.1038/s41567-019-0701-7 (2019).

2. Dong, G. \& Golden, S. S. Curr. Opin. Microbiol. 11, 541-546 (2008).

3. Nakajima, M. et al. Science 308, 414-415 (2005).

4. Strogatz, S. H. Physica D 143, 1-20 (2000). 